\title{
Evolution of $\mathrm{Pb}_{1-x} \mathrm{Cd}_{x} \mathrm{Te}$ Solid Solution Structure at High Temperatures
}

\author{
R. Minikayev ${ }^{a}$, E. Dynowska ${ }^{a}$, E. Kamińska ${ }^{b}$, A. Szczerbakow ${ }^{a}$, D. Trots $^{c, *}$, \\ T. STORY ${ }^{a}$ AND W. SzUszKIEWICZ ${ }^{a, *}$ \\ ${ }^{a}$ Institute of Physics, Polish Academy of Sciences, al. Lotników 32/46, 02-668 Warsaw, Poland \\ ${ }^{b}$ Department of Mathematics and Natural Sciences College of Science, Cardinal S. Wyszyński University \\ Dewajtis 5, 01-815 Warsaw, Poland \\ ${ }^{c}$ HASYLAB at DESY, Notkestr. 85, D-22603 Hamburg, Germany
}

\begin{abstract}
The recent successful growth of single, bulk $\mathrm{Pb}_{1-x} \mathrm{Cd}_{x} \mathrm{Te}$ crystals by self-selecting vapor transport method at the Institute of Physics of the Polish Academy of Sciences in Warsaw opened new opportunities to study the physical properties of this interesting material in detail. In this work we report the preliminary results of X-ray powder diffraction studies performed on a set of $\mathrm{Pb}_{1-x} \mathrm{Cd}_{x} \mathrm{Te}$ solid solutions (where $x \leq 0.056$ ) at high temperatures $(295 \mathrm{~K} \leq T \leq 1100 \mathrm{~K})$ and analyzed with the Rietveld refinement. Our results demonstrate the necessity of some correction of the relevant phase diagram and of the solubility limit, known from the literature.
\end{abstract}

PACS: $78.30 . \mathrm{Fs}, 81.10 . \mathrm{Fq}$

\section{Introduction}

The $\mathrm{PbTe}-\mathrm{CdTe}$ system in the compositional range on the $\mathrm{PbTe}$-rich side containing a lot of CdTe precipitates may be considered as a composite matrix perfectly matching principal properties required for future thermoelectric applications. However, it is difficult to obtain a uniform material of this kind. So far, in the case of bulk crystals grown by the Bridgman technique or by rapid quenching and annealing method only polycrystalline samples were obtained for the CdTe molar fraction $x>0.03$. For quenched $\mathrm{Pb}_{1-x} \mathrm{Cd}_{x} \mathrm{Te}$ solid solutions the phase diagram and, in particular, the solubility limit (close to $x \approx 0.03$ ) have been estimated experimentally long time ago [1-4], the thermodynamic behaviour of the $\mathrm{Cd}-\mathrm{Pb}-\mathrm{Te}$ system was analyzed by means of the phase diagram calculations based on previously published data [5]. Recently, the solubility limit of $\mathrm{Cd}$ in $\mathrm{PbTe}$ at $943 \mathrm{~K}$ have also been estimated in an independent manner [6].

The successful growth of single, bulk, metastable $\mathrm{Pb}_{1-x} \mathrm{Cd}_{x} \mathrm{Te}$ crystals (with $x \leq 0.11$ ) by self-selecting vapor transport method [7] at the Institute of Physics of the Polish Academy of Sciences in Warsaw opened new research opportunities. Due to their one-phase character

\footnotetext{
* Present address: Bayerisches Geoinstitut, Universität Bayreuth, Universitätsstr. 30, D-95447 Bayreuth, Germany.

* e-mail: szusz@ifpan.edu.pl
}

and the perfect structure quality, samples above mentioned were studied by several methods [8-10]. In particular, the preliminary results of our X-ray powder diffraction studies performed on several $\mathrm{Pb}_{1-x} \mathrm{Cd}_{x}$ Te solid solutions at high temperatures were shown in Ref. [9]. These results did not confirm the solubility limit known from the literature and suggested the necessity of some correction of the relevant phase diagram. Very recently, our findings were confirmed in part by the value of the solubility limit $x<0.01$ [11], which was much lower than that suggested previously.

In this work we report and discuss selected results of $\mathrm{X}$-ray powder diffraction studies performed with the use of synchrotron radiation on a set of $\mathrm{Pb}_{1-x} \mathrm{Cd}_{x} \mathrm{Te}$ solid solutions at high temperatures.

\section{Experimental details}

All crystals analyzed in this paper were grown by self-selecting vapor transport method [6] and characterized at the Institute of Physics, PAS in Warsaw. The one-phase character of samples was confirmed by the X-ray diffraction, taken on powdered crystals at room temperature using X'Pert Philips diffractometer and $\mathrm{Cu} K_{\alpha_{1}}$ radiation. The chemical composition of each sample was determined on the basis of their lattice parameter value $a$, using the relation $a(x)$, determined for the $\mathrm{Pb}_{1-x} \mathrm{Cd}_{x}$ Te solid solutions [8]. The crystals with $x=0$, $0.013,0.056$ were selected for further, detailed studies. The X-ray diffraction measurements were performed at the B2 beamline at Hasylab (DESY) using synchrotron 
radiation monochromatized at the photon wavelength $\lambda=0.5276 \AA$. The samples were prepared as a mixture of powdered $\mathrm{Pb}_{1-x} \mathrm{Cd}_{x}$ Te crystals and fine diamond powder (1:3 ratio). For sample mounting a thin-wall quartz capillary (rotating inside a graphite heater during the measurements) was applied. The diffraction patterns were accumulated when increasing temperature at several temperatures $(295 \mathrm{~K} \leq T \leq 1100 \mathrm{~K}$, the typical temperature step was equal to about $15 \mathrm{~K}$ ). After the measurement performed at highest applied temperature the sample was rapidly cooled down to about $900 \mathrm{~K}$, further slow decrease of temperature took several hours before reaching the ambient conditions. The temperature dependence of the lattice parameter $a$ was not analyzed when decreasing sample temperature, only the phase analysis and the chemical composition of the final solid solution were investigated at room temperature.

\section{Results and discussion}

For the analysis of the diffraction patterns the Rietveld method using the Fullprof.2k (v. 2.70) program was applied [12]. The example of the Rietveld refinement is shown in Fig. 1. At every temperature all structures observed in the pattern correspond to the Bragg reflections resulting from a presence of one $\mathrm{Pb}_{1-x} \mathrm{Cd}_{x} \mathrm{Te}$ solid solution crystal phase and the diamond powder only. The precise determination of the $\mathrm{Pb}_{1-x} \mathrm{Cd}_{x}$ Te lattice parameter values was possible due to the analysis of the diffraction patterns performed with the Rietveld refinement.

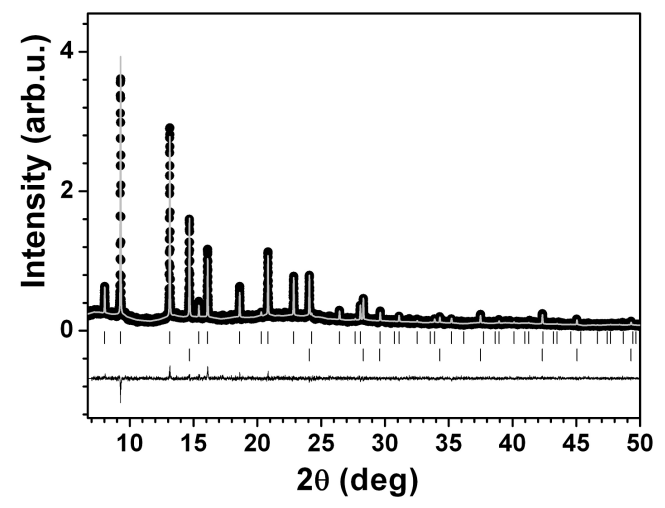

Fig. 1. Rietveld refinement of the structure for the sample containing $\mathrm{Pb}_{0.944} \mathrm{Cd}_{0.056}$ Te solid solution and the diamond powder. The powder diffraction pattern was obtained at $T=773 \mathrm{~K}$. Points: the experimental data taken with the use of synchrotron radiation, solid line: theoretical curve. The upper and the lower set of short vertical lines mark the positions of Bragg reflections corresponding to the $\mathrm{Pb}_{0.944} \mathrm{Cd}_{0.056} \mathrm{Te}$ crystal with the rock salt structure and to the diamond powder, respectively. The lowest curve shows the difference between observed and calculated powder diffraction patterns.

The results obtained in such a manner are presented in Fig. 2. As expected, the crystal lattice parameter at

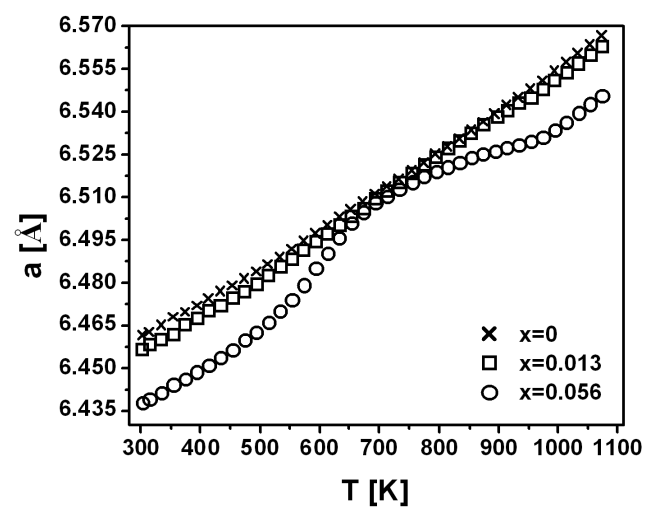

Fig. 2. The lattice parameter value versus temperature resulting from Rietveld refinement performed for investigated in this paper samples (for details see the discussion in text). The starting, room-temperature chemical compositions of $\mathrm{Pb}_{1-x} \mathrm{Cd}_{x} \mathrm{Te}$ solid solutions were equal to $x=0$ (crosses), $x=0.013$ (squares), and $x=0.056$ (circles).

room temperature decreases with an increasing $\mathrm{Cd}$ content in the solid solution. Moreover, the strong, nonlinear evolution of the lattice parameter with temperature for the sample containing before heating (at $295 \mathrm{~K}$ ) the $\mathrm{Pb}_{0.944} \mathrm{Cd}_{0.056} \mathrm{Te}$ solid solution is well seen in this figure. The change of the crystal chemical composition with temperature is the only explanation of this surprising effect. First, in the temperature range between $295 \mathrm{~K}$ and about $500 \mathrm{~K}$ the character of temperature evolution of the lattice parameter values determined for all investigated samples is quite similar. Within the experimental errors this evolution can be explained solely by the value of the expansion coefficient, corresponding to pure $\mathrm{PbTe}$ crystal and by the composition dependence of the lattice parameter at $295 \mathrm{~K}$. From temperature close to about $500 \mathrm{~K}$ investigated $\mathrm{Pb}_{0.944} \mathrm{Cd}_{0.056}$ Te solid solution starts to change its chemical composition. Between $500 \mathrm{~K}$ and $700 \mathrm{~K}$ the $\mathrm{Cd}$ content in analyzed crystal is reduced up to the composition slightly below $x=0.01$. This value matches perfectly the result given recently in Ref. [11]. The remaining $\mathrm{Cd}$ is still present in investigated sample but not as a constituent of the crystal phase (solid solution) but in the form of nanoclusters or small liquid droplets, which cannot be detected in the X-ray diffraction experiment (one has to mention that for bulk $\mathrm{Cd}$ the melting temperature is equal to $T=594 \mathrm{~K}$ but for $\mathrm{Cd}$ in the form of nanoclusters this temperature should be significantly smaller). The further increase of temperature results in an increase of the $\mathrm{Cd}$ content in $\mathrm{Pb}_{1-x} \mathrm{Cd}_{x} \mathrm{Te}$ solid solution and finally all $\mathrm{Cd}$ come back to the crystal phase forming once more a uniform $\mathrm{Pb}_{0.944} \mathrm{Cd}_{0.056} \mathrm{Te}$ solid solution. Similar behavior can be observed for the sample containing $\mathrm{Pb}_{0.987} \mathrm{Cd}_{0.013} \mathrm{Te}$, but the effect is not so spectacular in this case.

One has to remind that we have started our experiments with $\mathrm{Pb}_{1-x} \mathrm{Cd}_{x} \mathrm{Te}$ metastable solid solutions. 
Thus, in the temperature range between room temperature and $700 \mathrm{~K}$ we were dealing with metastable crystals and, in particular, their chemical compositions did not correspond to the relevant phase diagram. The thermodynamic equilibrium for investigated $\mathrm{Pb}_{1-x} \mathrm{Cd}_{x} \mathrm{Te}$ was reached at about $700 \mathrm{~K}$. The solid solution composition at $700 \mathrm{~K}$ ( $x$ slightly below 0.01$)$ corresponds to the solubility limit of $\mathrm{Cd}$ in $\mathrm{PbTe}$ at this temperature. Starting from $700 \mathrm{~K}$ with an increasing temperature one can investigate the phase diagram of the system under discussion.

Our results demonstrated in a direct manner that the $\mathrm{Pb}_{1-x} \mathrm{Cd}_{x}$ Te phase diagrams (where $x \leq 0.2$ ) or the numerical values corresponding to the solubility limit presented until now (see, e.g., [1-5]) do not describe correctly properties of the solid solution above mentioned and require a modification. According to our estimate, the correct solubility limit of $\mathrm{Cd}$ in $\mathrm{PbTe}$ at temperatures above $700 \mathrm{~K}$ is about half of that given in the literature and below this temperature it is close to $x=0.01$. Further X-ray diffraction studies are clearly required in order to complete the part of the phase diagram under consideration in the higher composition range and, in particular, to investigate the temporal evolution of $\mathrm{Pb}_{1-x} \mathrm{Cd}_{x} \mathrm{Te}$ structure properties when decreasing temperature from $1100 \mathrm{~K}$ to room temperature.

\section{Acknowledgments}

The research leading to these results has been partially supported by the European Community's Seventh Framework Programme (FP7/2007-2013) under grant agreement no. 226716, by the European Union within the European Regional Development Fund, through grant Innovative Economy (POIG.01.01.02-00-108/09), and by the Ministry of Science and Higher Education (Poland) grant DESY/68/2007.

\section{References}

[1] A.J. Rosenberg, R. Gierson, J.C. Woolley, P.M. Nikolic, Trans. Metall. Soc. A.I.M.E. 230, 342 (1964).

[2] P.M. Nikolic, Brit. J. Appl. Phys. 17, 341 (1966).

[3] A.J. Crocker, J. Mater. Sci. 3, 534 (1968).

[4] V. Leute, R. Schmidt, Z. Phys. Chem. 172, 81 (1991).

[5] Y. Liu, L. Zhang, D. Yu, J. Electron. Mater. 38, 2033 (2009).

[6] M.K. Sharov, J. Inorg. Chem. 55, 1119 (2010); Zh. Neorgan. Khim. 55, 1190 (2010) (in Russian).

[7] A. Szczerbakow, K. Durose, Prog. Cryst. Growth Character. Mater. 51, 81 (2005).

[8] M. Szot, A. Szczerbakow, K. Dybko, L. Kowalczyk, E. Smajek, V. Domukhovski, E. Łusakowska, P. Dziawa, A. Mycielski, T. Story, M. Bukała, M. Galicka, P. Sankowski, R. Buczko, P. Kacman, Acta Phys. Pol. A 116, 959 (2009).

[9] R. Minikayev, E. Dynowska, P. Dziawa, E. Kamińska, A. Szczerbakow, D. Trots, W. Szuszkiewicz, Synchr. Rad. Nat. Sci. 8, 83 (2009).

[10] B.A. Orlowski, A. Szczerbakow, B.J. Kowalski, M.A. Pietrzyk, K. Gas, M. Szot, W. Szuszkiewicz, V. Domukhovski, A. Reszka, S. Mickevicius, R.L. Johnson, S. Thiess, W. Drube, J. Electron Spectrosc. Relat. Phenom., in press.

[11] K. Ahn, M-.K. Han, J. He, J. Androulakis, S. Ballikaza, C. Uher, V.P. Dravid, M.G. Kanatzidis, J. Am. Chem. Soc. 132, 5227 (2010).

[12] J. Rodriguez-Carvajal, Newslett. IUCr Commission Powd. Diffr. 26, 12 (2001). 